\title{
Le retour du tramway dans les villes espagnoles : situation actuelle et perspectives.
}

Miguel Pazos Otón.

\section{(2) OpenEdition}

\section{Journals}

Édition électronique

URL : http://journals.openedition.org/rge/3524

DOI : $10.4000 /$ rge.3524

ISSN : 2108-6478

Éditeur

Association des géographes de l'Est

Édition imprimée

Date de publication : 15 juin 2012

ISSN : 0035-3213

Référence électronique

Miguel Pazos Otón., «Le retour du tramway dans les villes espagnoles : situation actuelle et perspectives. », Revue Géographique de l'Est [En ligne], vol. 52 / 1-2 | 2012, mis en ligne le 16 octobre 2012, consulté le 08 septembre 2020. URL : http://journals.openedition.org/rge/3524 ; DOI : https:// doi.org/10.4000/rge.3524

Ce document a été généré automatiquement le 8 septembre 2020

Tous droits réservés 


\title{
Le retour du tramway dans les villes espagnoles : situation actuelle et perspectives.
}

\author{
Miguel Pazos Otón.
}

\section{Introduction}

1 Lors de ces dernières décennies, le retour du tramway dans les villes espagnoles est un fait important dont les conséquences significatives réclament toute l'attention du géographe.

2 Au début du XXe siècle, la plupart des pays européens et l'Espagne en particulier comptaient un grand nombre de réseaux de tramways urbains qui furent démantelés peu à peu à partir des années soixante. On les remplaça par un système basé sur l'autobus. À la fin des années soixante-dix, le tramway disparaissait du paysage urbain des villes espagnoles. Trente ans plus tard, en 1984, Valence a suivi l'exemple de beaucoup de villes européennes et a mis en place un tramway moderne. Il y a actuellement douze villes espagnoles qui utilisent ce système de transport, ce qui constitue la confirmation du retour du tramway dans l'Espagne d'aujourd'hui.

3 Le tramway est un mode de transport qui a de nettes implications territoriales. D'une part, il résout des problèmes de déplacement quotidien de la population dans des espaces de plus en plus saturés de voitures et où l'embouteillage est devenu un des principaux problèmes; et d'autre part, il aide à créer une nouvelle image urbaine, plus moderne et dynamique devenant un élément essentiel dans les politiques de rénovation urbaine (CHIGNIER-RIBOULON, 2003).

4 Nous voulons analyser ici dans un premier temps la chronologie de la nouvelle implantation du tramway dans les zones urbaines et métropolitaines espagnoles. Nous présenterons ensuite le chapitre central du travail : un classement des systèmes de tramways existant en Espagne d'après leur fonctionnalité territoriale, autrement dit, le rapport qu'il y a avec le territoire. En conclusion, nous réfléchirons synthétiquement 
sur l'importance des rapports entre le tramway et le territoire dans l'Espagne d'aujourd'hui.

\section{LE RETOUR DU TRAMWAY EN ESPAGNE}

5 L'importance de la présence actuelle du tramway en Espagne a des antécédents historiques. C'est à la fin du XIXe siècle (à Madrid en 1871) que commença l'implantation de ce système de transport dans les villes espagnoles. On passa de la traction animale à la traction mécanique et, en particulier, électrique. En 1899, Barcelone est la première espagnole qui adopte un tramway électrique.

6 Vers la moitié du XXe siècle, le tramway s'imposa comme moyen de transport hégémonique. Les villes espagnoles purent s'étendre et croître grâce au tramway qui permettait la mobilité quotidienne des ouvriers des banlieues aux zones centrales industrielles (MIRAS, 2001).

7 Dans les années cinquante et soixante, le tramway disparait au profit du trolleybus. Ce processus fut similaire et parallèle (bien que différé dans le temps) à celui que subirent les réseaux de tramway des États-Unis où les intérêts des entreprises de caoutchouc eurent un rôle essentiel. Le trolleybus fut très vite remplacé par l'autobus qui fut généralisé dans les années soixante-dix (TARTAJO, 2006).

8 En 1975, c'est à Saragosse (TARTAJO, 2006) que le dernier tramway cesse de circuler. L'augmentation de la mobilité motorisée donnait une image d'encombrement dans les zones urbanisées. C'est ce qu'avait anticipé BUCHANAN dans son célèbre rapport «Traffic in towns» (1964). En 1990, Torres et al. écrivaient: «(...) on assiste ces dernières années à une dégradation évidente des conditions de transport et de circulation dans les principales villes espagnoles (...)»(TORRES et al, 1990). La situation empirait d'année en année.

9 De notre point de vue, ce phénomène (qui est toujours très actuel) obéit à plusieurs motifs parmi lesquels l'efficacité limitée des municipalités dans le contrôle de la localisation des activités économiques; nous assistons aussi à l'apparition et à la généralisation de la dénommée "ville diffuse ", "la ville des voies périphériques encombrées" (sic HALL (1996) qui implique la diffusion de l'urbanisation dans un modèle de faibles densités résidentielles dans le territoire urbain périphérique (suburbain, dans la littérature anglo-saxonne).

10 Il faut donc encadrer le retour du tramway dans ce contexte. En 1994, Valence est la première ville espagnole qui décide de réimplanter le tramway. Il existe un vrai retard historique par rapport au contexte européen car l'inauguration du premier tramway moderne espagnol a eu lieu neuf ans après celle de son homologue en France, celui de Nantes.

11 Huit ans plus tard, en 2002, a lieu le lancement du deuxième nouveau tramway espagnol, à Bilbao. À partir de là, les implantations de ce moyen de transport se succèdent rapidement: Alicante (2003), Barcelone (2004), Vélez-Málaga (2006), Tenerife, Madrid, Vitoria, Parla, Murcie et Séville (2007) et Saragosse (2011). La figure 1 montre la distribution spatiale des tramways dans le territoire espagnol. 
Figure 1 : Distribution spatiale des tramways dans le territoire espagnol

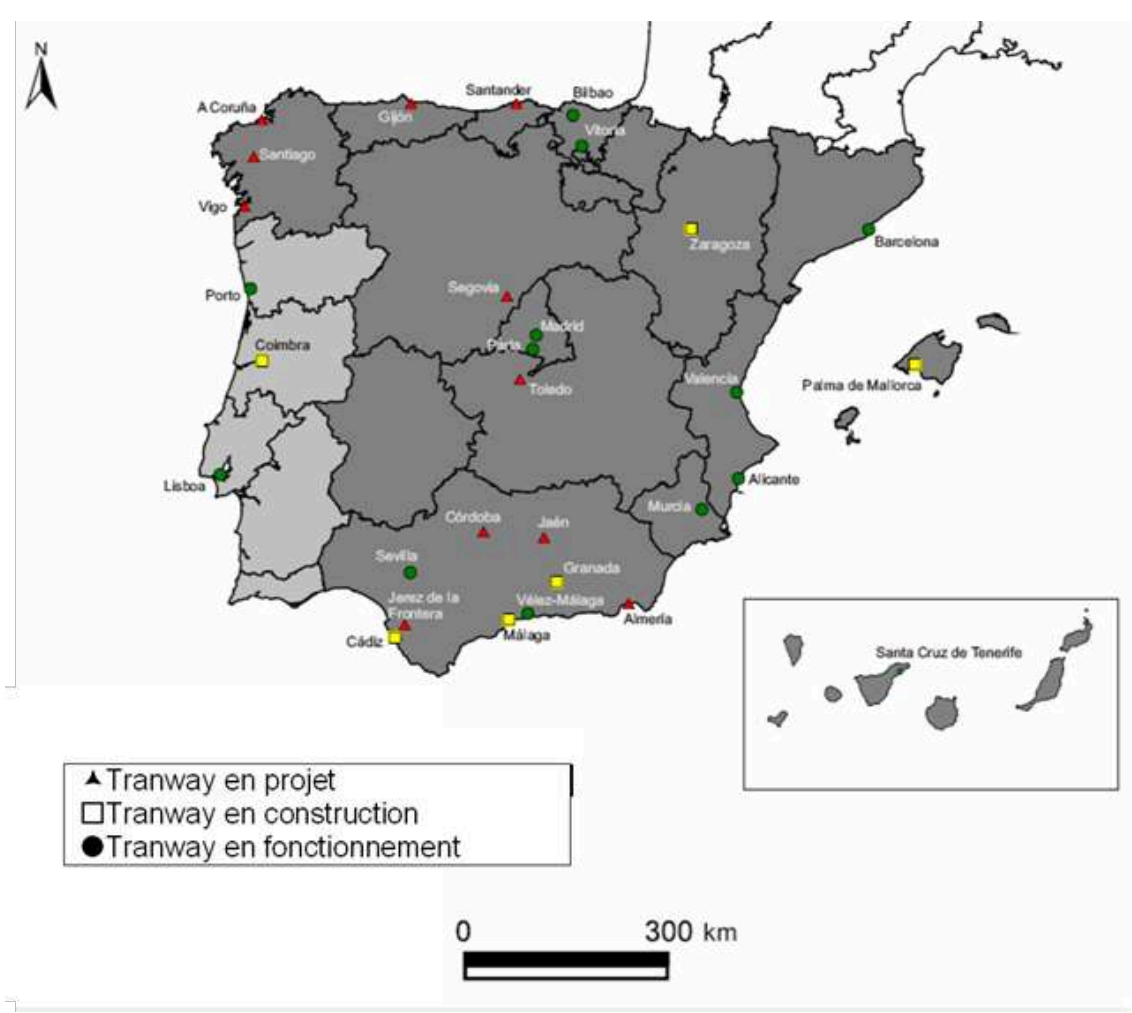

12 On peut remarquer dans cette carte que les systèmes de tramways sont concentrés de préférence sur les espaces littoraux par rapport à une faible présence dans la zone intérieure. Madrid, Parla et Saragosse sont d'ailleurs les seules villes de toutes les provinces intérieures espagnoles qui possèdent un tramway. Ce fait est dû non seulement à la concentration de la population espagnole sur la frange côtière mais aussi au dynamisme économique et social des périphéries espagnoles : le Pays Basque et le littoral Méditerranéen sont des espaces gagnants dans le contexte géo-économique espagnol actuel.

13 Nous allons nous concentrer à présent sur les tramways en fonctionnement et analyser leur implantation dans les espaces de référence urbains et régionaux. Pour approfondir notre analyse, nous ferons une proposition de classement des tramways selon leur fonctionnalité territoriale.

\section{LES TRAMWAYS ESPAGNOLS ACTUELS D'APRÈS LEUR FONCTIONNALITÉ : UNE PROPOSITION DE CLASSEMENT.}

14 Les tramways présentent une certaine hétérogénéité par rapport au territoire qu'ils desservent et les fonctions qu'ils réalisent. Un tramway peut être un moyen de transport exclusivement urbain mais il peut être aussi métropolitain et régional. L'Espagne possède trois types de tramway et nous pouvons en ajouter une quatrième par sa singularité : les tramways touristiques.

15 Nous avons établi ici comme cadre d'analyse un classement qui examine les traits principaux des tramways actuels. 


\subsection{Tramways urbains} compacte et ils ont une fonctionnalité semblable à celle d'un bus urbain, connectant des points de mobilité assez proches (plusieurs centaines de mètres) dans un paysage urbain qui ne présente pas de discontinuités.

Cette typologie présente néanmoins des difficultés pour marquer les limites de la ville compacte. Nous avons donc appliqué ce critère avec une certaine relativité par rapport aux limites administratives des communes. Ce type de tramways entre dans un réseau multimodal et joue un rôle efficace de connecteur entre des terminaux résolvant ainsi des problèmes de connectivité du réseau général. Il circule en général à travers des circuits «nobles» ou emblématiques de la ville (par exemple: Avenue Diagonal à Barcelone, etc).

\section{a) Le tramway de Bilbao}

Bilbao est un des berceaux de l'industrialisation espagnole. Cette ville comptait sur un réseau important de tramways lors des vingt premières années du XIXe siècle. En 1920, il faisait plus de cent kilomètres et possédait douze lignes qui partaient du centre de Bilbao (VIA LIBRE, 2009).

De nos jours, après Valence, Bilbao est la deuxième ville d'Espagne qui a un tramway moderne inauguré en 2002 sous la marque nationale Euskotran. Cet empressement dans le développement et la mise en place du projet à Bilbao s'explique essentiellement par la coopération et la concertation entre les différents niveaux d'administrations. La société Bilbao Ría 2000 a été créée en vue de stimuler un grand projet de rénovation urbaine. Pour ce faire, il y a eu l'établissement d'un accord entre le gouvernement basque et le gouvernement municipal de Bilbao puisque les deux appartiennent au même parti politique, le PNV (« Parti Nacionalista Vasco »).

On a voulu assainir les rives du fleuve Nervión et transformer une ville industrielle en crise en un grand centre de services moderne avec un élément catalyseur : le Musée Guggenheim situé sur des terrains d'anciens chantiers navals et d'entrepôts industriels (JUARISTI, 2003).

21 Parallèlement à la transformation d'Abandoibarra et à la construction du Guggenheim, Bilbao Ría 2000 a projeté le tramway moderne en suivant le cours du fleuve et a recherché nettement une image de rénovation et de modernité face à la dégradation pré-existante.

Le tramway a été envisagé comme une priorité par les administrations publiques locale et régionale pour contribuer à une opération de rénovation urbaine et améliorer l'image dégradée de Bilbao. Cette ville est le symbole du Pays Basque basée jadis sur la sidérurgie et l'industrie lourde. Elle entra en crise au début des années 1980 avec la reconversion du secteur naval et sidérurgique. Le caractère nationaliste des deux administrations citées explique le caractère symbolique et la priorité attribuée au projet qui prétendait incarner, avec le Musée Guggenheim, l'image d'une nouvelle région basque, Euskadi, ouverte vers l'extérieur et pleinement contemporaine.

Le mémoire correspondant à l'année 2009 publié par Euskotran (voir tableau 1) apporte des données significatives d'exploitation. 
Tableau 1 : Données significatives du tramway de Bilbao (2009)

\begin{tabular}{|l|l|}
\hline Nombre de kilomètres & 310.844 \\
\hline Nombre de places & 8.121 .250 \\
\hline Nombre de voyageurs & 2.799 .452 \\
\hline
\end{tabular}

Source: Euskotram. Mémoire de l'année 2009

Le tableau démontre le bon comportement de ce tramway qui a transporté 2.800 .000 voyageurs en 2009. Il faut tenir compte toutefois du nombre de places offertes qui a dépassé 8.000 .000 et ceci se traduit par une occupation relative de $34,4 \%$. Pour ce qui est de l'ensemble des tramways espagnols que nous traiterons plus tard, il s'agit d'une position intermédiaire que l'on peut expliquer par le fait que le tramway ne dessert que la ville compacte de Bilbao et ses nouveaux quartiers. Pour la banlieue, on utilise le Métro et les trains de banlieue de la RENFE.

Dans la pratique, le tramway fait communiquer deux points de mobilité importants : le Musée Guggenheim et San Mamés avec la gare centrale intermodale de Abando et El Arenal, point d'union entre la Vieille Ville et les nouveaux quartiers de la ville. Le tramway n'est donc pas utilisé de la même façon pour la mobilité quotidienne que pour des voyages ponctuels (visites touristiques, les jours de matchs de football, etc).

\section{b) Le tramway de Vitoria-Gasteiz}

À Vitoria-Gasteiz, la capitale du Pays Basque, l'idée du tramway date de la deuxième moitié des années 1990, au moment où le Gouvernement basque présenta un projet à la Municipalité alors qu'il rencontrait une forte opposition citoyenne. Plus tard, l'opinion publique est revenue sur sa décision à partir d'une exposition en 2002 intitulée «Vitoria bouge » qui aida à apprécier les aspects positifs du tramway. 
Figure 2 : Le tramway circulant dans la Calle General Álava

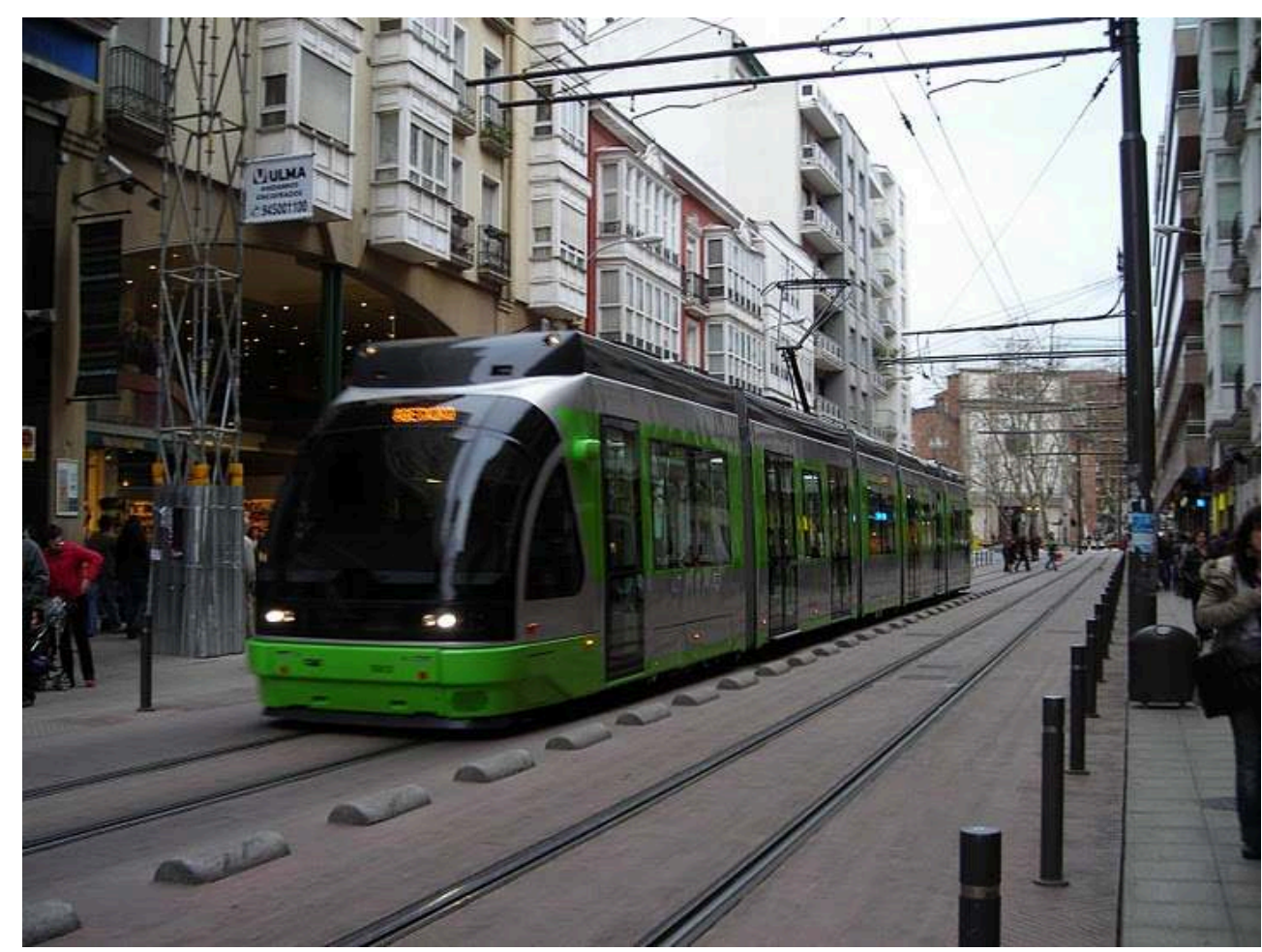

Auteur: Miguel Pazos Otón. Date: 20.03.2010

27 Tout comme à Bilbao, la coopération et la concertation entre les différents niveaux d'administration eut une très grande importance dans le projet de création du tramway de Vitoria-Gasteiz. Le gouvernement du Pays Basque, le gouvernement provincial de Álava et le gouvernement municipal ont accepté de financer conjointement le projet. La plus grande contribution a été celle du Gouvernement Basque avec $65 \%$ du total.

28 En 1964, on approuva le projet du circuit du tramway au coût approximatif de cent millions d'euros et un parcours de neuf kilomètres. Un des aspects les plus remarquables dans la planification de ce projet de transport fut sa connexion avec les plans d'urbanisme généraux de la ville. Le tracé des nouvelles lignes de tramway a tenu compte du Plan général de l'Aménagement municipal et en particulier de la construction de nouveaux quartiers de la ville.

29 La figure 3 montre un panneau d'indication avec le plan du tramway dans la ville. On peut remarquer le tracé de la voie du Chemin de fer, au sud, connectée au départ du réseau du tramway. 
Figure 3 : Panneau d'indication avec le plan du tramway à Vitoria-Gasteiz.

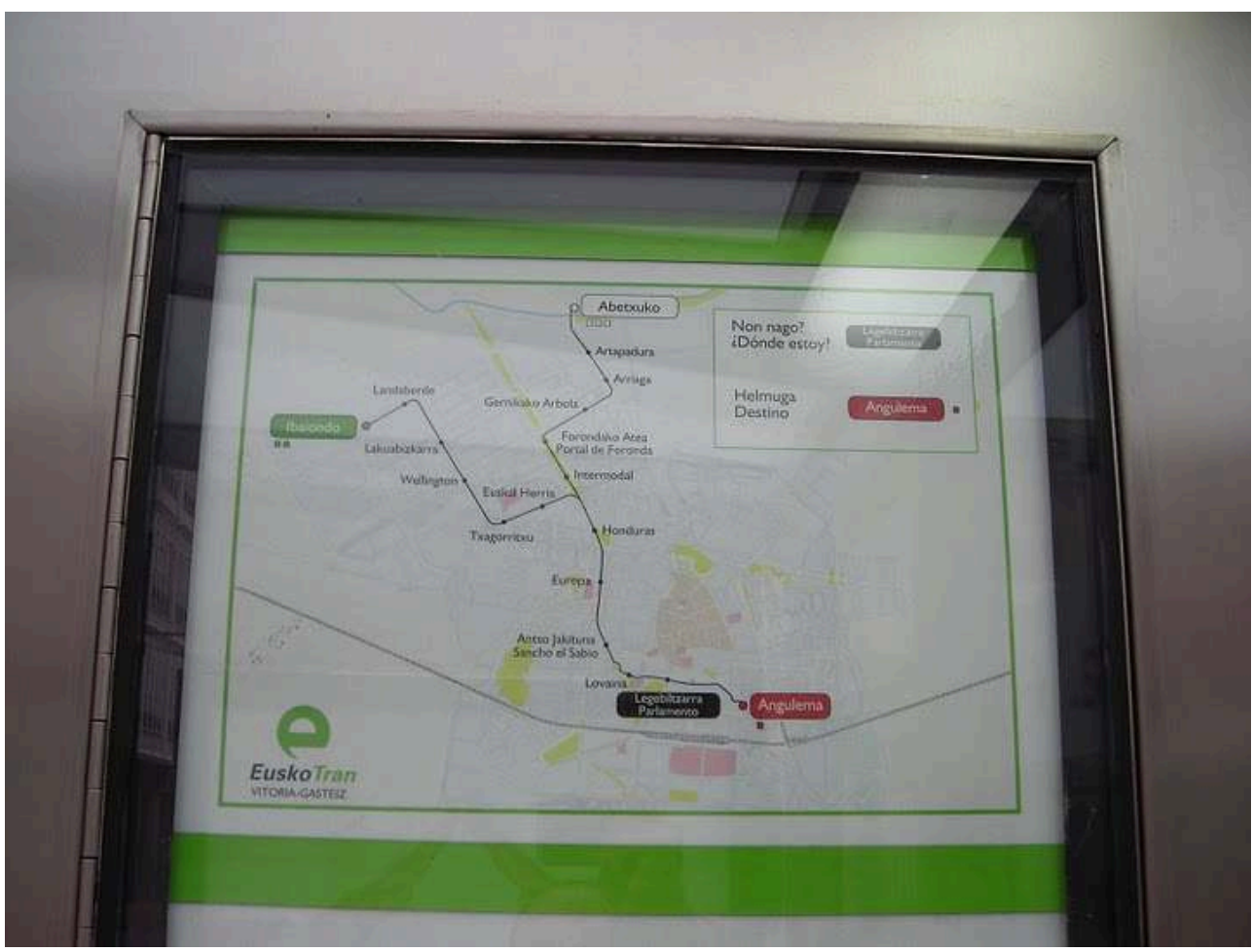

Auteur : Miguel Pazos Otón. Date 20.03.2010

Au départ de la gare ferroviaire, les deux lignes de tramway ont un tracé commun qui traverse le cœur du centre-ville en passant par le siège du Parlement basque et très près de la Plaza de la Virgen Blanca, en plein centre de Vitoria-Gasteiz. Le tracé du tramway se poursuit vers le nord et bifurque avec une ligne vers le terminal Ibaiondo et une autre ligne vers Abetxuko. Ces deux terminus sont justement les fronts du progrès de l'urbanisation de la ville et le tramway. Celui-ci garantit une bonne accessibilité aux nouveaux développements urbains. Nous pouvons parler pour ainsi dire d'un TransitOriented Design ou d'une planification urbaine étroitement liée à la dotation de transport.

31 Les travaux, dirigés par l'entreprise publique EuskoTran, ont commencé en septembre 2006 et le tramway fut inauguré le 23 décembre 2008. Depuis lors, l'expérience du tramway de Vitoria s'est avéré être un vrai succès. En deux ans de fonctionnement (voir tableau 1) on a dépassé 11 millions de voyageurs pour un parcours total d'environ 800.000 kilomètres (EUSKOTREN, 2011).

Les chiffres correspondants au nombre d'usagers ci-dessous révèlent nettement la réussite du tramway à Vitoria-Gasteiz (voir tableau 2).

Tableau 2 : Statistiques de voyageurs du tramway de Vitoria-Gasteiz (2009)

\begin{tabular}{|l|l|}
\hline Moyenne de passagers par jour ouvré & 12.120 \\
\hline Moyenne de passagers par jour férié & 8.500 \\
\hline
\end{tabular}


Moyenne mensuelle de passagers

305.000

Source : Annuaire du Chemin de Fer 2010 « Fundación de los Ferrocarriles Españoles »

33 La proportion entre les voyages réalisés les jours ouvrés et les jours fériés démontre que le tramway est non seulement utilisé dans les déplacements obligatoires quotidiens mais aussi dans les habitudes générales de la population.

Des données plus récentes publiées dans la rubrique des nouvelles de la page web de Euskotran révèlent qu'en novembre 2009 et en novembre 2010 le tramway de Vitoria a connu une augmentation de $10,23 \%$.

Il y a une satisfaction générale du service de la part des usagers. Dans une enquête menée par Euskotran auprès des usagers, ce mode de transport a obtenu 8,4 sur 10 pour sa ponctualité et 8,04 pour sa propreté et son confort.

Les bénéfices du tramway ne se limitent pas à l'amélioration de la mobilité et de l'accessibilité. Dans l'enquête citée, neuf usagers sur dix ont déclaré que le tramway a contribué à l'amélioration de l'image de modernité de la ville. Ce qui confirme l'hypothèse que le tramway est non seulement un mode de transport mais aussi un instrument de rénovation urbaine.

37 L'inauguration en juillet 2009 de la deuxième tranche affermit ce système de transport urbain nouveau très apprécié des citadins. Le tramway a favorisé le caractère central de la «Plaza de la Virgen Blanca » et l'élimination de la circulation dans beaucoup de rues de la ville. Il canalise de même la croissance de nouveaux axes de développement en le connectant au centre ville d'une façon plus efficace. Finalement, dans sa mise en place, il y a eu un vrai souci esthétique qui est mis en évidence dans le design des abribus et du matériel roulant ou dans l'aménagement de jardins dans l'espace du tramway dans les zones périphériques.

\section{c) Le tramway de Séville: « Metrocentro »}

Le tramway de Séville a été inauguré en 2007 sous la dénomination "Metrocentro ». Actuellement, la ligne 1 du tramway de Séville est un train métropolitain aux caractéristiques d'un métro léger. Tout comme les précédents, le tracé de la ligne est conçu pour favoriser l'inter- modalité. Il existe ainsi des connexions avec le Métro de Séville et les services de trains de banlieue.

Le circuit actuel est «Plaza Nueva-San Bernardo». Le caractère métropolitain du tramway est très présent grâce à la connexion avec le Métro de Séville et surtout avec les réseaux aux noyaux satellites de l'agglomération de la banlieue sévillane de Dos Hermanas, Alcala de Guadaira, La Rinconada et El Aljarafe.

\section{d) Le tramway de Saragosse}

40 Saragosse est une ville très compacte et aux densités de population fort élevées. Lors de ces dernières années, elle a recherché sans équivoque à améliorer sa mobilité et a entrepris un Plan de Mobilité Durable. Un des objectifs a été de transformer Saragosse en une ville modèle dans la politique de mobilité. C'est ainsi que l'on a favorisé la complémentarité entre les différents moyens de transport sans pour autant omettre l'importance de la participation publique. 
41 Le Plan se rapporte aussi à l'importance du respect de l'esthétique et des valeurs traditionnelles malgré les changements introduits dans la ville. C'est une raison pour laquelle le tramway convient tout à fait puisque il cohabite avec la circulation routière. Il a été inauguré en avril 2011 avec la ligne 1 entre Valdespartera et le Parque Goya. Le «Tranvía de Zaragoza » est géré par la « Sociedad de Economía Mixta de los Tranvías de Zaragoza " à laquelle participent également le Municipalité de Saragosse, TUZSA (Société de Transports Urbains de Saragosse), CAF (société de matériaux ferroviaires), Ibercaja (société d'épargne locale) ainsi que trois autres entreprises du bâtiment. Nous avons là un consortium public et privé, une formule de gestion moderne qui s'impose de plus en plus dans les infrastructures et les services de transports de tout type.

Le 22 septembre 2011, après cinq mois à peine de service, le tramway de Saragosse obtient le Prix «Semana de la Mobilidad Sostenible » (Semaine de la Mobilité Durable) organisé par le Ministère de l'Environnement et du Milieu rural et marin, pour ses mesures innovatrices en faveur de la mobilité durable. Nous pouvons souligner la promotion de l'inter modalité tramway et bicyclette et du véhicule électrique grâce à l'installation de points de recharge dans les gares urbaines d'interconnexion des modes de transports.

\section{e) Le tramway de Madrid et de Parla}

Dans la Communauté autonome de Madrid (Région de Madrid) existent quatre lignes de tramway appelées «métros légers de Madrid». Elles furent toutes inaugurées en 2007. Elles desservent des zones à forte population (espaces urbains compacts) rendant possible l'échange modal entre des autobus et des trains de banlieue.

Les trois premières lignes de tramway lient entre elles diverses stations du Métro de Madrid en trajets transversaux. Pour ce qui est de la quatrième qui a 8,2 kilomètres, elle se développe entièrement dans la commune de Parla (banlieue de Madrid) et constitue un défi particulier de la commune à titre individuel bien qu'elle soit intégrée dans l'ensemble du réseau et dans le système de transports métropolitains de Madrid.

Le tableau 3 montre un volume de voyageurs mensuel de 340.000 qui représente un volume annuel de plus de quatre millions et qui dépasse largement le chiffre de Bilbao (près de trois millions).

Tableau 3 : Statistiques de voyageurs du tramway de Parla

\begin{tabular}{|l|r|}
\hline Moyenne de passagers par jour ouvré & 17.800 \\
\hline Moyenne de passagers par jour férié & 8.260 \\
\hline Moyenne mensuelle de passagers & 340.000 \\
\hline
\end{tabular}

Source : Annuaire des Chemins de fer 2010 «Fundación de los Ferrocarriles Españoles »

Quatre ans après sa mise en place, les estimations réalisées expriment des points de vue différents. La municipalité (qui n'a plus le même maire qui avait lancé le tramway bien que l'actuel soit du même parti politique) offrent les chiffres de voyageurs et son 
augmentation annuelle comme étant une preuve de succès. De son côté, l'opposition critique le coût élevé pour Parla pour l'entretien du tramway.

Le quotidien $A B C$ va au-delà et publie le 12 juillet 2011 «Le projet pharaonique de Gómez (le maire précédent de Parla et promoteur du tramway) sur le point de disparaître à cause de la dette ». Le 30 novembre 2011, le tramway de Parla cessa son activité puisque la Municipalité devait 48 millions d'euros à la société concessionnaire Tranvías S.A qui à son tour devait 6,5 millions d'euros à Alstom, la société chargé de la maintenance. À l'origine de ce problème, il y a les différences idéologiques entre le Conseil municipal de Parla et le gouvernement régional de Madrid.

Le tramway de Parla fut inauguré en 2007. La Municipalité a apporté $87 \%$ du coût; de son côté, le gouvernement régional n'y a investi que 13\%. Durant toutes ces années, le Conseil municipal a accusé la Communauté régionale de discriminer le tramway de Parla en lui attribuant un appui financier inférieur à ceux d'autres communes madrilènes à même idéologie politique que le gouvernement régional. Celui-ci s'est défendu en alléguant qu'il aurait été moins cher et plus efficace de mettre en œuvre un système d'autobus.

50 Le 1er décembre 2011, sous la pression du Maire de Parla devant le gouvernement régional, un accord créditeur a été conclu. On a établi ainsi un calendrier de paiements et la société Alstom cessa ses prestations. Le tramway de Parla reprit son activité ce jour même.

\subsection{Tramways métropolitains}

51 Ce deuxième groupe de tramways est caractérisé par un service desservant des espaces périphériques. Ils ont une fonction semblable à un autobus métropolitain ou à un train de banlieue. La dimension totale des réseaux est de plusieurs dizaines de kilomètres. Il peut y avoir des arrêts séparés de plus d'un kilomètre.

52 Ces tramways sont organisés dans des espaces métropolitains et ils traversent des paysages urbains qui n'ont pas forcément de continuité en édifices. Ils font communiquer souvent le centre-ville avec une série de cités satellites ou périphériques et avec l'aéroport. Il s'agit là d'un domaine supra municipal.

\section{a) Le tramway de Valence : «Metrovalencia »}

53 C'est le premier tramway moderne construit en Espagne. Il fut inauguré en 1994 avec une ligne qui unit des points-clés de mobilité tels les pôles universitaires et la Plage de la Malvarrosa. Il récupère le tracé de l'ancien tramway qui allait à la Malvarrosa.

54 Après plusieurs inaugurations de lignes, le tramway arrive aujourd'hui jusqu'à l'aéroport de Manises dans la ceinture périphérique de Valence. La vocation métropolitaine du système est significative dans le projet de construction de la ligne de la Huerta Sur (L'Horta Sud). Cette nouvelle ligne prévoit une mobilité interne pour un ensemble de communes bien reliées à Valence. C'est un système radial à travers le réseau de trains de banlieue ou d'autobus métropolitains.

Le système de tramway de Valence entre dans le cadre d'une région, la Communauté Valencienne (Région de Valence) victime de gros problèmes territoriaux qui ont nui et détérioré son image. La construction démesurée et la colonisation frénétique du littoral ont conduit à une situation d'un énorme impact environnemental et écologique. La 
grande partie de la population espagnole voit là un littoral "malade» qui á été « enseveli par le ciment ». multimodal de transports de la zone métropolitaine. Il connecte la gare ferroviaire et les gares de trains de banlieue. La Connexion avec l'aéroport est très estimable et va contribuer à relancer une infrastructure de transport qui se trouvait jusque là nettement sous-employée. Le tableau 4 montre les statistiques d'usagers dans le tramway de Valence. Il reflète le poids important du nombre de voyageurs en jours ouvrés qui est le double de celui des jours fériés.

Tableau 4: Statistiques de voyageurs du tramway de Valence

\begin{tabular}{|l|r|}
\hline Moyenne de passagers par jour ouvré & 32.000 \\
\hline Moyenne de passagers par jour férié & 15.000 \\
\hline Moyenne de passagers par mois & 450.000 \\
\hline Moyenne de passagers par an & 5.300 .000 \\
\hline
\end{tabular}

Source : Annuaire du Chemin de Fer 2010 «Fundación de los Ferrocarriles Españoles »

Nous devons souligner aussi le nombre de voyageurs annuels, plus de cinq millions. C'est un volume élevé non seulement par la taille de l'agglomération de Valence mais aussi par l'importance des points de mobilité qu'il connecte et par la tradition dans l'utilisation du tramway par la population (rappelons qu'il fut mis en place en 1994).

\section{b) Le tramway de Barcelone}

59 Le réseau de tramways de Barcelone est intégré dans les dénommés TramBaix et TramBesòs et le tracé central de l'Avenida Diagonal. Ce schéma est conçu pour fournir une mobilité aux noyaux satellites de Hospitalet, Cornellà, Esplugues, San Just Desvern et San Joán Despí et Sant Adriá de Besós (à l'ouest).

60 Le renouveau du tramway moderne s'est produit dans le contexte d'une Barcelone après les jeux Olympiques de 1992 même si les premières études réalisées par la EMT (Entitat Metropolitana del Transport) datent de 1987. Cette année-là, la Generalitat (le gouvernement régional) a supprimé la Zone Métropolitaine de Barcelone parce qu'il y voyait un contre-pouvoir qui pouvait rivaliser avec le gouvernement régional.

61 La création de la AMT (Autoritat Metropolitana del Transport) en 1998 a un format de Consortium de Transports métropolitains. Elle a permis de surmonter les divergences entre le gouvernement catalan et les municipalités concernées. Il convient de préciser que dans la Zone Métropolitaine de Barcelone il y avait une majorité de municipalités gouvernées par le Parti Socialiste de Catalunya tandis que dans la Generalitat le pouvoir était de Convergència i Unió (parti nationaliste). 

Barcelona, on a inauguré la dernière ligne ouverte aujourd'hui à la circulation: TramBesòs. grandes raisons.

Tout d'abord, tel qu'il est indiqué dans le tableau 5, il y a un très grand nombre d'usagers au mois, plus de 1.300 .000 . Ceci implique plus de 15 millions de voyageurs par an et plus de cinq fois le volume de passagers de Bilbao.

Tableau 5 : Statistiques du tramway de Barcelone

\begin{tabular}{|l|r|}
\hline Moyenne de passagers par jour ouvré & 56.300 \\
\hline Moyenne de passagers par jour férié & 14.800 \\
\hline Moyenne mensuelle de passagers & 1.305 .000 \\
\hline
\end{tabular}

Source : Annuaire des Chemins de fer 2010 «Fundación de los Ferrocarriles Españoles »

Remarquons le grand développement du réseau du tramway de Barcelone et la communication entre les noyaux résidentiels de la périphérie de Barcelone et le centreville. La plus grande partie de la mobilité du tramway de Barcelone se produit pour des motifs de travail ou d'études, c'est-à-dire quotidiennement. Ceci est vérifiable puisque les jours fériés la mobilité est quatre fois moins grande que les jours ouvrés (cf. tableau $5)$.

D'autre part, l'importance du tramway de Barcelone est due à l'image de modernité et de rénovation urbaine que montre la "Avenida Diagonal », un des axes principaux de mobilité de la ville. Le passage du tramway sur cet itinéraire «noble » où se trouvent les sièges de la plupart des grandes banques catalanes, reflète l'intention de favoriser l'image de Barcelone comme symbole, capitale de la Catalogne et le sentiment nationaliste accru comme au Pays Basque.

\section{c) Le tramway de Tenerife}

67 Dans les Iles Canaries, en particulier dans l'Ile de Tenerife, se trouve le seul tramway existant dans un archipel du territoire espagnol (excepté le tramway touristique de Sóller, à Majorque). Le tramway a une vocation nettement métropolitaine puisqu'il se développe tout le long du corridor Santa Cruz de Tenerife-La Laguna, et il parcourt un espace à caractère nettement touristique.

Il s'agit de deux villes de 250.000 et 100.000 habitants respectivement séparées d'à peine dix kilomètres. Santa Cruz de Tenerife est la capitale de la province; c'est une ville portuaire et commerciale. Tandis que La Laguna (Patrimoine de l'Humanité) est une ville touristique et universitaire.

69

Cette différence explique l'existence d'une forte mobilité quotidienne (pour le travail ou les études), d'une mobilité pour achats, déplacements pour des motifs médicaux, etc. et bien sûr de la mobilité touristique.

Revue Géographique de l'Est, vol. 52 / 1-2 | 2012 
70 L'histoire de ce tramway remonte au début du XXe siècle puisque c'est en 1901 que fut inauguré un tramway pour joindre les deux villes mais avec un circuit différent.

71 À la fin du XXe siècle, le gouvernement insulaire («Cabildo ») souhaita récupérer l'idée d'un tramway entre les deux pôles les plus importants de cette agglomération. Il se heurta à une forte opposition politique et de la population mais malgré tout le projet fut lancé. Aujourd'hui le tramway résout une bonne partie des problèmes de déplacement d'un espace urbain complexe où l'utilisation de l'automobile est encore importante.

\section{d) Le tramway de Vélez-Malaga}

72 Ce premier tramway pour la région d'Andalousie fut inauguré en 2006. Il se trouve dans la zone de la Axarquia (à l'est de Malaga). Il unit la ville intérieure de Vélez-Málaga de 75.000 habitants et Torre del Mar (d'environ 25.000 habitants), un noyau côtier caractérisé par l'importance touristique.

Ces deux zones forment un espace urbain bipolaire d'environ 100.000 habitants. C'est pour cette raison que ce tramway entre dans la typologie des tramways métropolitains.

Après deux ans de fonctionnement, et comme dans le cas du tramway de Parla, celui de Vélez-Málaga était clairement déficitaire. Avec une dette accumulée de 53 millions d'euros en novembre 2011, on décida l'interruption de son fonctionnement puisque ni le Conseil municipal ni le gouvernement régional ne pouvaient assumer les frais. Après plusieurs mois d'attente, le 26 avril 2012, on décréta la suspension de la circulation du tramway pour début juin 2012.

Tout comme pour le cas de Parla, nous sommes face à la négligence municipale qui s'est embarquée dans des projets trop onéreux qui ne comptaient nullement sur l'appui nécessaire dans les autres niveaux des administrations publiques. Dans la situation de crise économique actuelle, face à l'existence d'une grande quantité de dettes, la seule solution est la fermeture des services de tramway. Ceci signifie le gaspillage d'une grande quantité d'argent public.

\subsection{Systèmes tram-train : le tramway régional. Le tram-train d'Alicante.}

Les systèmes tram-train constituent une nouveauté récente en Espagne. Comme son nom l'indique, il s'agit de l'intégration de deux modes de transport très proches: le tramway et le chemin de fer conventionnel. Les tramways sont alors considérés comme un équipement technique noble qui permet la circulation aussi bien par la voie ferrée conventionnelle que la voie ferrée classique. La double capacité de voltage permet au tram-train d'avoir un comportement de train de banlieue et de tramway urbain.

Cette adaptabilité du tram-train lui permet de parcourir des distances beaucoup plus longues que celles des systèmes basés simplement sur le tramway. On tire profit des voies ferrées conventionnelles pour aller au-delà du réseau métropolitain et desservir un espace sous-régional. En Espagne il n'existe qu'un seul tram-train qui se trouve à Alicante. Il dessert la quasi-totalité du littoral de la province du même nom, d'Alicante jusqu’à Dénia. 
78 Le littoral d'Alicante appelé "Costa Blanca » est un espace à très forte vocation touristique. On y trouve Benidorm, un des pôles du soleil et sa célèbre plage connue dans le monde entier. Nous avons là une offre très large et une fréquentation touristique durant toute l'année, ce qui crée une demande de mobilité constante. La Costa Blanca est manquée en outre par une grande occupation de l'espace littoral avec de grands centres touristiques consolidés tels : Altea, Calpe-Calp, Jávea-Xábia ou Dénia qui cohabitent avec un grand réseau de zones résidentielles à caractère secondaire.

79 C'est dans ce contexte qu'il est facile de justifier la mise en place d'un système tramtrain intégré qui permet le déplacement en transport collectif à travers toute cette zone de résidences. Les bénéficiaires en sont les riverains mais aussi les touristes. La Compagnie concessionnaire TRAM édite même des brochures touristiques pour découvrir le littoral d'Alicante en tramway.

\subsection{Tramways touristiques}

80 Il existe un autre type de tramways appelés «tramways touristiques » qui correspond tout à fait à ce que PAGE (2005) dénomme «transport for tourism » puisqu'il s'agit de systèmes de transport qui aujourd'hui offrent avant tout une attraction touristique.

81 Ils s'inspirent de modèles de tramways du début du XXe siècle, recréant une ambiance d'époque qui propose au touriste un voyage dans le temps tout en admirant l'espace urbain. Il n'existe aujourd'hui en Espagne que le tramway de Sóller car le service de celui de La Corogne a cessé depuis peu. Nous y reviendrons ci-dessous.

82 Le tramway de Sóller (ville côtière de l'île de Majorque) fut inauguré en 1913 faisant suite au train qui unissait Palma de Majorque et Sóller. Il y a moins de cinq kilomètres de distance entre Sóller e le «Puerto de Sóller ». La raison de son existence est due à l'impossibilité de faire passer les voies ferrées conventionnelles par le centre de cette localité.

83 Le tramway fut conçu en principe pour des passagers mais au cours du temps il servit aussi au transport des marchandises (poisson et charbon en particulier). Il fonctionna tout le long du XXe siècle s'attribuant de plus en plus une dimension touristique. C'est aujourd'hui un tramway comme dans un théâtre où la découverte et le plaisir du paysage ont un rôle essentiel. Les wagons conservent une forme fidèle, l'ambiance de l'époque du début du XXe siècle, ce qui est un plus dans cette expérience de voyage touristique.

84 Pour revenir au tramway de La Corogne déjà mentionné plus haut, il a cessé de circuler, depuis quelques mois, mais il faut le citer car c'est une des rares expériences de tramway touristique d'Espagne.

85 Il était apparu dans les années 1990 en parallèle avec la construction de la promenade maritime urbaine qui contourne la péninsule où se trouve la ville. À la fin des travaux, les responsables municipaux souhaitèrent récupérer un des anciens tramways historiques (en particulier un wagon de tramway qui faisait la ligne entre A Coruña et Sada) pour créer une ligne qui fasse le tour de la péninsule de La Corogne.

86 Ce fut une grande attraction touristique pour la ville : on pouvait faire la visite des alentours de la Tour d'Hercule (Monument Patrimoine de l'Humanité), longer la Promenade Maritime de Riazor et d'Orzan où se trouvent les deux plages les plus fréquentées. 


\section{Conclusions et perspectives d'avenir} prospère dans notre pays. De nombreuses villes ont misé sur ce mode de transport et d'autres vont le faire dans les prochaines années. Le gouvernement régional de Malaga, par exemple, stimule le Métro de Malaga et les travaux sont bien avancés. En Andalousie, on est en train de mettre en œuvre des systèmes de tramway et de métro léger sur la Baie de Cadix (agglomération urbaine polycentrique) ainsi qu'à Grenade. Aux Îles Baléares, Palma de Majorque est en travaux pour mettre en place le tramway. Trois grandes villes galiciennes ont élaboré des projets d'installation du tramway: Vigo, La Corogne et Saint-Jacques de Compostelle. Au nord de la Péninsule, Gijon et Santander, villes maritimes, ont également en vue une rénovation urbaine avec ce mode de transport.

D'autres cas où le tramway est en phase de projet sont les villes desservies par le train à grande vitesse (AVE : alta velocidad española); en effet, c'est le cas de Tolède et de Ségovie où les gares de l'AVE présentent des localisations périphériques. Le tramway permettrait d'améliorer l'accès aux gares et de mieux structurer le déplacement urbain. 


\section{BIBLIOGRAPHIE}

BUCHANAN, C.D. (1964): Traffic in Towns (Repport of the Department of Transport, UK, 1963), Penguin Books, London.

CHIGNIER-RIBOULON, F. (2003): Large housing estates in France: overview of developments and problems in Lyon. Faculty of Geosciences, Utrecht University. Utrecht

HALL, P. (1996): Ciudades del mañana. Historia del urbanismo en el siglo XX. Ediciones del Serbal, Colección La Estrella Polar. Barcelona.

JUARISTI, J. (2003): El patrimonio histórico-industrial y la revitalización urbana y económica de Bilbao. Boletín del Instituto Andaluz del Patrimonio Histórico, Año oํ 11, № 42, 2003 , págs. 79-87. Junta de Andalucía. Sevilla.

MIRÁS ARAÚJO, J. (2001): El tranvía como vehículo de configuración urbana. La localización de la actividad económica en la ciudad de A Coruña durante la primera mitad del XX. Comunicación presentada al II Congreso de Historia Ferroviaria, con el titulo de "Siglo y medio de ferrocarriles en Madrid". Aranjuez, Febrero de 2001.

PAGE, S. (2005): Transport and Tourism. Pearson Education. Harlow, UK.

TARTAJO GARRIDO, J.A. (2006): El ocaso de los tranvías españoles. Comunicación presentada al IV Congreso de Historia Ferroviaria. Málaga, Septiembre de 2006.

TORRES, J., RODRÍGUEZ BAYRA y BARCELÓ, J. (1990): "Políticas de transporte, tráfico y circulación: cómo articular los diferentes modos de transporte", en J. Borja, M. Castells, R. Dorado e I. Quintana (Eds.), Las grandes ciudades en la década de los novnta, pp. 409-427. Ed. Sistema. Madrid.

\section{Références Internet}

Factoría Urbana : http://www.factoriaurbana.com

Tranvías de Zaragoza: web oficial http://www.tranviasdezaragoza.com

Vía libre. La revista del ferrocarril (2009): Informe de tranvías y metros ligeros en España. http:// www.vialibre-ffe.com/noticias.asp?not=2928\&cs=oper 


\section{RÉSUMÉS}

Lors de ces dernières années, on assiste au retour du tramway dans les villes espagnoles. Depuis 1994, il élargit l'offre de modes de transports urbains en donnant une meilleure mobilité et accessibilité et en rénovant l'image des villes. Cet article donne une vision générale de la situation du tramway en Espagne à partir d'une proposition de classement fonctionnelle basée sur des tramways urbains, métropolitains, régionaux et touristiques. La situation actuelle et l'avenir de ce transport, qui verra le jour dans plusieurs villes, feront l'objet d'une analyse.

In recent years there has been the return of the tram in Spanish cities. Since 1994, it increased the supply of urban transport modes by providing greater mobility and accessibility and renovating the image of cities. This article gives an overview of the situation of the tram in Spain from a proposed classification based on functional urban, metro, regional and touristic tram. The current situation and future of this transport, which will be launched in several cities will be analyzed.

INDEX

Keywords : tramway, return, Spain, urban, metropolitan, mobility

Mots-clés : tramway, retour, Espagne, urbain, métropolitain, mobilité

\section{AUTEUR}

\section{MIGUEL PAZOS OTÓN.}

Département de Géographie. Université de Santiago de Compostela. Facultade de Xeografía e Historia. Praza da Universidade, 1. E-15782 Santiago de Compostela (Espagne). e-mail: miguel.pazos.oton[chez]usc.es 